To appear in Recent Progress in Many-Body Theories, vol. 4, edited by E. Schachinger, et al. (Plenum, New York).

\title{
IMPROVED TREATMENT OF FREQUENCY SUMS IN PROPAGATOR-RENORMALIZED PERTURBATION THEORIES
}

\author{
J.J. Deisz ${ }^{a}$, D.W. Hess ${ }^{b}$, and J.W. Serene ${ }^{a}$ \\ ${ }^{a}$ Department of Physics \\ Georgetown University \\ Washington, D.C. 20057-0995 \\ ${ }^{b}$ Complex Systems Theory Branch \\ Naval Research Laboratory \\ Washington, D.C. 20375-5000
}

\section{Introduction}

This work stems from calculations for Hubbard [1, 2, 3] and Anderson lattice [4, 5] models in a self-consistent conserving Green's function scheme [6, 7] known as the the fluctuation exchange approximation (FEA) [8]. For the 2D Hubbard model, special features of band structure, such as Fermi surface nesting [9] and van Hove singularities near the Fermi surface [10, 11], lead to anomalous frequency and momentum dependences of the self-consistent self-energy [3, [12]. At half filling the FEA self-energy develops a frequency dependence similar to that proposed for a marginal Fermi liquid [13], and the spin-fluctuation propagator appears to move exponentially close to an instability with decreasing temperature. When the spin-fluctuation propagator is sufficiently close to this instability, we have been unable to obtain stable converged solutions. For the halffilled 3D Hubbard model, where an antiferromagnetic phase transition is expected at finite temperature, we have studied the fully self-consistent spin response to a staggered magnetic field [14]. The results are qualitatively similar to those in 2D [15], and show no magnetic order for a range of $U$ and $T$ well within the antiferromagnetic phase expected from Quantum Monte Carlo simulations [16].

For the Anderson lattice model, we have observed the evolution of a coherent quasiparticle state with decreasing temperature along with a substantial enhancement in the effective mass [4]. As in the Hubbard model, for parameters relevant to correlated electronic systems, the spin fluctuation propagator is very near an instability at low 
temperatures. We would like to calculate reliably the entropy and specific heat as a function of temperature, to elucidate the apparent transformation from a lattice of local moments in a sea of 'ordinary' conduction electrons to a band of highly renormalized quasiparticles.

The only numerical approximation (other than finite machine precision) in our previous implementations of the FEA has been the treatment of the high-frequency tails of Green's functions, self-energies, etc. Although we are primarily interested in understanding the low-energy excitations, high-energy processes make important contributions to effective masses, susceptibilities, total energies, etc., and numerical approximations in treating these processes must be controlled, and so far as possible eliminated, to obtain reliable results for the problems described above. In this paper we describe a new approach to handling high-frequency tails. We decompose the single particle Green's function into two parts,

$$
G\left(\mathbf{k}, \varepsilon_{n}\right)=g\left(\mathbf{k}, \varepsilon_{n}\right)+\tilde{G}\left(\mathbf{k}, \varepsilon_{n}\right),
$$

where, as we will make more precise below, $\tilde{G}$ contains only 'low' frequency parts of $G$ and $g$ contains the leading 'high' frequency parts (through some order). The important observation is that relatively little information is contained in the high frequency tails of the Green's function. The crucial trick is to find an analytic form for $g$ that describes the high frequency behavior of $G$ accurately and leads to tractable analytic expressions for the contributions from $g$ to susceptibility bubbles, $T$-matrices and self-energies. Most of the detailed information about correlations resides in $\tilde{G}$, which is much less sensitive to the frequency cutoff than was the original $G$. In this language, most previous approaches to solving the FEA numerically correspond to taking $g$ to be identically zero [17].

Taking advantage of massively parallel computers requires scalable algorithms that perform efficiently for a wide range of problem sizes using virtually any number of processors. To this end, our algorithm solves the equations of the FEA iteratively, making use of discrete Fourier transforms at various stages of the calculation to make each step embarrassingly parallel. To motivate our new approach, we first sketch a less accurate but more straightforward way to calculate the FEA self-energy.

\section{Standard Implementation of the Fluctuation Exchange Approximation}

Central to propagator renormalized perturbation theory is Dyson's equation, relating the renormalized propagator $G$ to the self-energy $\Sigma$,

$$
G^{-1}\left(\mathbf{k}, \varepsilon_{n}\right)=G_{0}^{-1}\left(\mathbf{k}, \varepsilon_{n}\right)-\Sigma\left(\mathbf{k}, \varepsilon_{n}\right),
$$

where $G_{0}$ is the Green's function of the non-interacting system. For simplicity we will discuss only the simplest paramagnetic Hubbard model, and will include in the selfenergy only the proper second-order diagram and the contribution from exchanged spin fluctuations; including the density and pairing fluctuations is straightforward and involves no new matters of principle. The fluctuation propagator is constructed from a susceptibility bubble which is a convolution product of renormalized propagators,

$$
\chi_{p h}\left(\mathbf{q}, \omega_{m}\right)=-\frac{T}{N} \sum_{\mathbf{k}, n} G\left(\mathbf{k}+\mathbf{q}, \varepsilon_{n}+\omega_{m}\right) G\left(\mathbf{k}, \varepsilon_{n}\right) .
$$


In terms of $\chi_{p h}$ the fluctuation propagator ( $T$-matrix) is simply

$$
T\left(\mathbf{q}, \omega_{m}\right)=\frac{3}{2}\left[\frac{U \chi_{p h}\left(\mathbf{q}, \omega_{m}\right)^{2}}{1-U \chi_{p h}\left(\mathbf{q}, \omega_{m}\right)}\right],
$$

and the self-energy is a convolution of the Green's function with the sum of the $T$-matrix and the susceptibility bubble,

$$
\Sigma\left(\mathbf{k}, \varepsilon_{n}\right)=U^{2} \frac{T}{N} \sum_{\mathbf{q}, \omega_{m}} G\left(\mathbf{q}+\mathbf{k}, \omega_{m}+\varepsilon_{n}\right)\left[\chi_{p h}\left(\mathbf{q}, \omega_{m}\right)+T\left(\mathbf{q}, \omega_{m}\right)\right] .
$$

To obtain the self-consistent self-energy, one starts with a guess for $G\left(\right.$ e.g. $\left.G_{0}\right)$ and calculates the self-energy from Eqs.(3-5). The resulting $\Sigma$ is then used in Dyson's equation to update the propagator, and this procedure is iterated to some level of approximate self-consistency.

The sums in these equations extend over all momenta and all frequencies, but in a numerical calculation we can include only a finite number of terms. For the momentum sums this is simply equivalent to taking a finite lattice with periodic boundary conditions. Truncating the frequency sums admits no simple physical interpretation, however, and providing an alternative to truncation is the focus of this paper. From the perspective of this section, the most straightforward procedure is to introduce a sharp cutoff, by setting the Green's function, self-energy, etc. equal to zero for all $\left|\varepsilon_{n}\right|>\varepsilon_{c}$, which we will call the sharp cut-off scheme. This leads to highly artificial behavior of the susceptibility, $T$-matrix, and self-energy with increasing frequency. More important from the point of view of instabilities and phase transitions, the self-energies and susceptibilities at low frequencies lose high-frequency contributions from Green's functions, susceptibilities, and T-matrices.

\section{Posing the Problem Another Way}

The fluctuation-exchange approximation for the Hubbard model (and related lattice models such as the Anderson lattice model) has a special feature that makes it especially well-suited for a fine-grained SIMD parallel computer such as the Connection Machine: the bare interaction is completely local in space and time, and the approximation does not introduce any nonlocal effective interactions. As a result, all equations of the theory can be solved completely in parallel at each point in either $\left(\mathbf{k}, \varepsilon_{n}\right)$ space (Dyson's equation and the $T$-matrix equations) or $(\mathbf{r}, \tau)$ space (susceptibilities and self-energy),

without the need to evaluate directly any convolutions. In the $(\mathbf{r}, \tau)$ representation, the susceptibility bubble is simply

$$
\chi_{p h}(\mathbf{r}, \tau)=-G(\mathbf{r}, \tau) G(-\mathbf{r},-\tau)
$$

and the self-energy is

$$
\Sigma(\mathbf{r}, \tau)=U^{2}\left[\chi_{p h}(\mathbf{r}, \tau)+T_{p h}(\mathbf{r}, \tau)\right] G(\mathbf{r}, \tau),
$$

while the natural representations of the T-matrix and Dyson equation are given by Eqs. (2) and (4) above. From this point of view, a possible approach is to begin from 
$G_{0}\left(\varepsilon_{n}\right)$ with a sharp high-frequency cutoff as before, and to transform back and forth between $\left(\mathbf{k}, \varepsilon_{n}\right)$ and $(\mathbf{r}, \tau)$ using fast Fourier transforms (FFTs); we will call this the $\varepsilon_{n^{-}}$ scheme. This yields Green's functions at a discrete set of evenly spaced $\tau$-points between 0 and $\beta$, but the sharp cutoff causes endpoint ringing near $\tau=0$ and $\tau=\beta$. Because many physical quantities come from the Green's function at precisely these endpoints, a better approach is to begin the the calculation from the exact $G_{0}(\tau)$ sampled on a uniform mesh of $\tau$-points, which, after an FFT, has the effect of introducing a gentle high-frequency cutoff in $G_{0}\left(\varepsilon_{n}\right)$. The previous calculations described in the introduction use this approach, which we will call the $\tau$-scheme [3].

\section{Contributions from High-Frequency Parts of the Green's Function}

Repeated integration by parts of the Fourier integral for $G\left(\mathbf{r}, \varepsilon_{n}\right)$ shows that the discontinuities of the Green's function and its derivatives at $\tau=0$ determine the high-frequency behavior of the Green's function,

$$
G\left(\mathbf{r}, \varepsilon_{n}\right)=\frac{-\Delta G(\mathbf{r})}{i \varepsilon_{n}}+\cdots+\frac{(-1)^{p+1} \Delta G^{(p)}(\mathbf{r})}{\left(i \varepsilon_{n}\right)^{p+1}}+\frac{(-1)^{p+1}}{\left(i \varepsilon_{n}\right)^{p+1}} \int_{0}^{\beta} e^{i \varepsilon_{n} \tau} \frac{\partial^{p+1} G(\mathbf{r}, \tau)}{\partial \tau^{p+1}} d \tau,
$$

where

$$
\left.\Delta G^{(p)}(\mathbf{r}) \equiv \frac{\partial^{p} G(\mathbf{r}, \tau)}{\partial \tau^{p}}\right|_{\tau=0^{+}}-\left.\frac{\partial^{p} G(\mathbf{r}, \tau)}{\partial \tau^{p}}\right|_{\tau=0^{-}}
$$

Substituting this expression for the Green's functions and a similar high-frequency expansion for $\Sigma$ into Dyson's equation leads to expressions for the discontinuities of the renormalized propagator in terms of unrenormalized single-particle energies $\xi_{\mathbf{k}}$ and discontinuities in $\Sigma$,

$$
G\left(\mathbf{k}, \varepsilon_{n}\right) \sim \frac{1}{i \varepsilon_{n}}+\frac{\xi_{\mathbf{k}}+\Sigma_{H}}{\left(i \varepsilon_{n}\right)^{2}}+\frac{\left(\xi_{\mathbf{k}}+\Sigma_{H}\right)^{2}-\Delta \Sigma(\mathbf{k})}{\left(i \varepsilon_{n}\right)^{3}}+\ldots
$$

where $\Sigma_{H}$ is the Hartree-Fock contribution to the self-energy. For the Hubbard model with nearest-neighbor hopping only, Eq. (10) gives the first two discontinuities as

$$
\Delta G(\mathbf{r})=-\delta_{\mathbf{r}, \mathbf{0}}, \quad \Delta G^{\prime}(\mathbf{r})=(-\mu+U n / 2) \delta_{\mathbf{r}, \mathbf{0}}-t \delta_{|\mathbf{r}|, 1},
$$

where $\mu$ is the chemical potential, $t$ is the hopping matrix element, $n$ is the (selfconsistent) density and $U$ is the Hubbard interaction.

We write $G$ as the sum of an analytic part $g\left(\mathbf{r}, \varepsilon_{n}\right)$ containing the leading highfrequency behavior, and a part $\tilde{G}\left(\mathbf{r}, \varepsilon_{n}\right)$ represented numerically up to a maximum frequency $\varepsilon_{c}$,

$$
G\left(\mathbf{r}, \varepsilon_{n}\right)=\tilde{G}\left(\mathbf{r}, \varepsilon_{n}\right)+g\left(\mathbf{r}, \varepsilon_{n}\right) .
$$

A simple analytic form for $g\left(\mathbf{r}, \varepsilon_{n}\right)$ that includes the discontinuities of Eq. (11) is

$$
g\left(\mathbf{r}, \varepsilon_{n}\right)=-\Delta G(\mathbf{r}) Q_{0}\left(\varepsilon_{n}, x_{0}(\mathbf{r})\right)+\Delta G^{\prime}(\mathbf{r}) Q_{1}\left(\varepsilon_{n}, x_{1}(\mathbf{r})\right)
$$

with

$$
Q_{0}\left(\varepsilon_{n}, x_{0}(\mathbf{r})\right)=\frac{1}{2}\left[\frac{1}{i \varepsilon_{n}-x_{0}(\mathbf{r})}+\frac{1}{i \varepsilon_{n}+x_{0}(\mathbf{r})}\right] \rightarrow \frac{1}{i \varepsilon_{n}} \quad \text { for } \varepsilon_{n} \rightarrow \infty
$$




$$
Q_{1}\left(\varepsilon_{n}, x_{1}(\mathbf{r})\right)=\frac{-1}{2 x_{1}(\mathbf{r})}\left[\frac{1}{i \varepsilon_{n}+x_{1}(\mathbf{r})}-\frac{1}{\left.i \varepsilon_{n}-x_{1}(\mathbf{r})\right)}\right] \rightarrow \frac{1}{\left(i \varepsilon_{n}\right)^{2}} \quad \text { for } \varepsilon_{n} \rightarrow \infty .
$$

The discontinuity and derivative discontinuity of $G$ are included in $g$ independent of the choices for $x_{0}(\mathbf{r})$ and $x_{1}(\mathbf{r})$. We choose these parameters by setting $\tilde{G}(\mathbf{r}, 0)$ for $|\mathbf{r}|=0,1$ and $\tilde{G}^{\prime}(\mathbf{0}, 0)$ equal to zero; we show below that this choice is optimal when forming the second-order self-energy.

In Fig. (11) we illustrate this decomposition of the non-interacting Green's function in both $\tau$ and $\varepsilon_{n}$ space. The solid curve is the full $G_{0}$, the dashed line is $G_{0}-Q_{0}$, and and the dotted line shows the final numerical part, $\tilde{G}_{0}=G_{0}-Q_{0}+\mu Q_{1}$, which is represented on a discrete $\tau$-mesh and transformed with an FFT. The smoother and smaller $\tilde{G}_{0}$ has a spectral weight that is effectively confined to low frequencies so that the errors introduced by $\varepsilon_{c}$ are much smaller than those incurred in an FFT of the full Green's function in any of the standard cutoff schemes. Analytic terms are Fourier transformed exactly and functional forms keep track of contributions to infinite frequency.
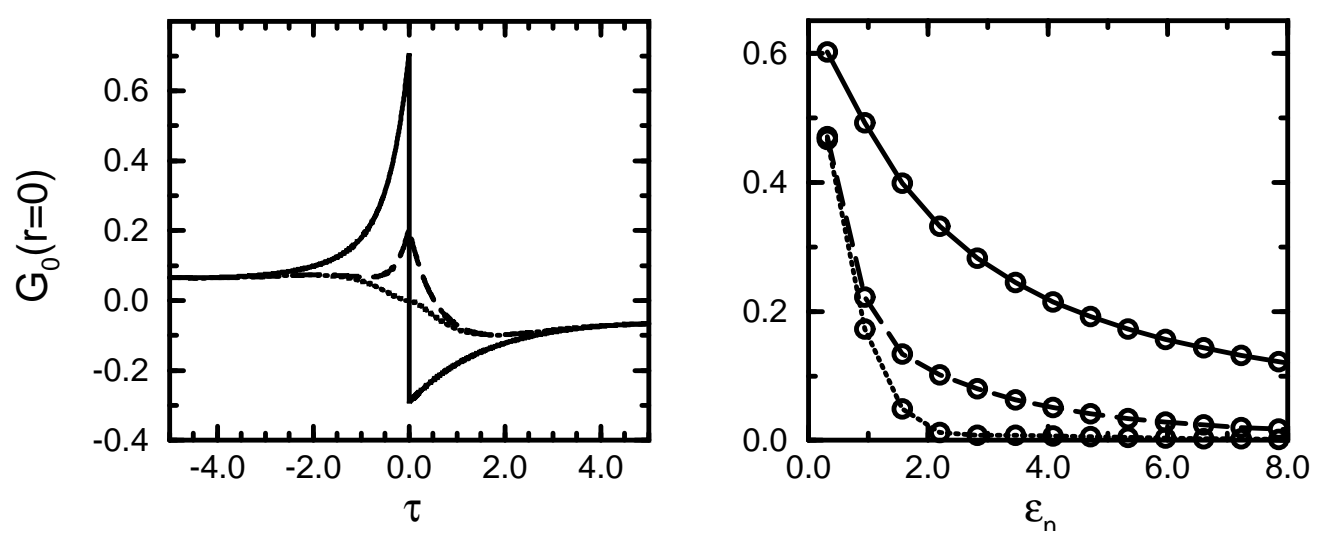

Figure 1: The non-interacting Green's function $G_{0}$ at $r=0$ as a function of $\tau$ (left) and its modulus as a function of $\varepsilon_{n}$ (right) for a 1D Hubbard model for $T=0.1 t$ and $\mu=1.2 t$. The solid curve is $G_{0}(r=0)$, the dashed curve is $G_{0}(r=0)-Q_{0}$, and the dotted line is $\tilde{G}(r=0)=G_{0}(r=0)-Q_{0}+\mu Q_{1}$. The removal of discontinuities at $\tau=0$ in the part of $G_{0}$ represented numerically corresponds to removing high-frequency tails in $\varepsilon_{n}$-space; $\tilde{G}_{0}\left(\varepsilon_{n}\right)$ has nearly vanished for frequencies greater than a bandwidth $W=4 t$. Note that all energies are measured in units of the hopping matrix element $t$.

As described earlier, the FFT method takes advantage of the relative simplicity of expressions like that for the second-order self-energy in $(\mathbf{r}, \tau)$ space,

$$
\Sigma_{2}(\mathbf{r}, \tau)=-U^{2} G(\mathbf{r}, \tau) G(\mathbf{r}, \tau) G(-\mathbf{r},-\tau) .
$$

Using our decomposition of $G$, a part of this expression can be calculated analytically,

$$
\sigma_{2}(\mathbf{r}, \tau)=-U^{2} g(\mathbf{r}, \tau) g(\mathbf{r}, \tau) g(-\mathbf{r},-\tau)
$$

since this consists of simple functions of $\tau$ with analytic Fourier transforms. We choose $x_{0}(\mathbf{r})$ and $x_{1}(\mathbf{r})$ so that $\sigma_{2}(\mathbf{r}, \tau)$ contains the leading discontinuities of $\Sigma(\mathbf{r}, \tau)$. This 
choice is optimal in that the remaining numerical piece is continuous to second order at $\tau=0$. For example, the leading discontinuity in $\Sigma_{2}(\mathbf{r}, \tau)$ is given by

$$
\begin{aligned}
\Delta \Sigma_{2}(\mathbf{r}) & \equiv \Sigma_{2}\left(\mathbf{r}, 0^{+}\right)-\Sigma_{2}\left(\mathbf{r}, 0^{-}\right) \\
& =-U^{2} G\left(\mathbf{0}, 0^{-}\right) G\left(\mathbf{0}, 0^{+}\right) \Delta G(\mathbf{r})
\end{aligned}
$$

which is finite only for $\mathbf{r}=\mathbf{0}$. Since $x_{0}$ and $x_{1}$ are chosen such that $\tilde{G}(\mathbf{0}, 0)$ vanishes, this discontinuity is contained entirely in $\sigma_{2}(\mathbf{r}, \tau)$,

$$
\Delta \Sigma_{2}(\mathbf{r})=-U^{2} g\left(\mathbf{0}, 0^{-}\right) g\left(\mathbf{0}, 0^{+}\right) \Delta g(\mathbf{r}) \equiv \Delta \sigma(\mathbf{r}) .
$$

In Fig. (2), we show $\Sigma_{2}$ calculated with $G=G_{0}$ as a solid curve and the numerical part, $\Sigma_{2}-\sigma_{2}$, with a dotted curve. It is only this numerical function whose Fourier transform is approximated with an FFT. As shown in Fig. (3) this leads to a large reduction in error in the self-energy in $\left(\mathbf{k}, \varepsilon_{n}\right)$ space at all frequencies in comparison to the errors found with the sharp cut-off, $\tau^{-}$, and $\varepsilon_{n}-$ schemes.

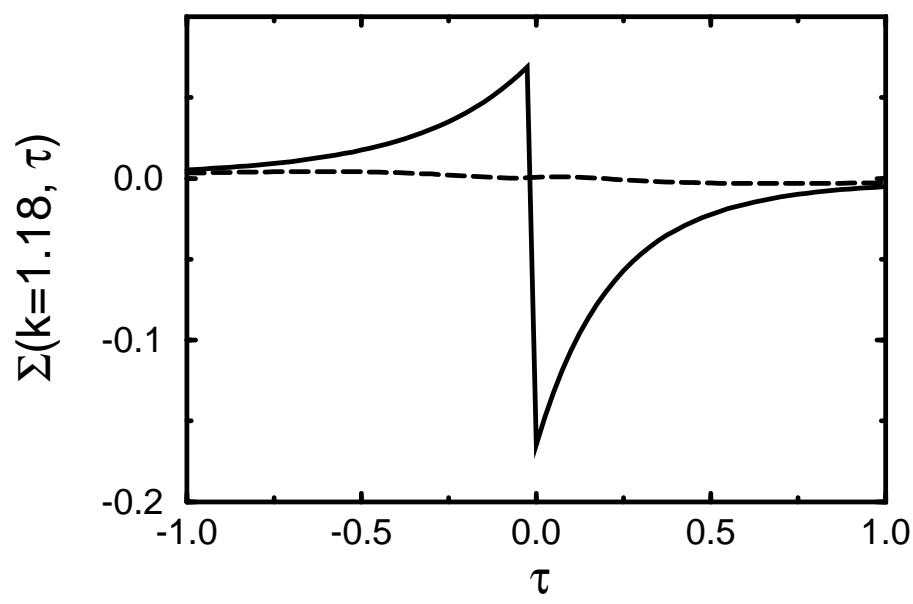

Figure 2: Full second-order self-energy (solid) calculated with the bare propagator $G_{0}$ for a $1 \mathrm{D}$ Hubbard model with $U=t, \mu=-0.7 t$ and $T=0.04 t$ and the the numerical part (dashed) obtained by subtracting an analytic contribution evaluated using $g$.

In a self-consistent calculation, the optimal parameters are adjusted iteration by iteration as correlations change the $\tau \rightarrow 0$ values of the Green's function and its derivatives. In Fig. (4) we show the $\varepsilon_{0}$ point of the self-consistent second-order self-energy as a function of the number of points kept in the representation for $\tilde{G}$ compared to that obtained with the $\tau$-scheme. As this figure illustrates, the improvements realized in Fig. (3) are also realized in a self-consistent calculation. In particular, as shown for the self-energy at its lowest frequency, $\varepsilon_{0}=\pi T$, substantially fewer points are required for this method to achieve the infinite frequency cut-off limit (obtained with, say, the $\tau$-scheme) to acceptable accuracy.

It is possible to achieve results with higher accuracy and more rapid convergence by adding more analytic terms in $g(\mathbf{r}, \tau)$ to include higher-order derivative discontinuities 

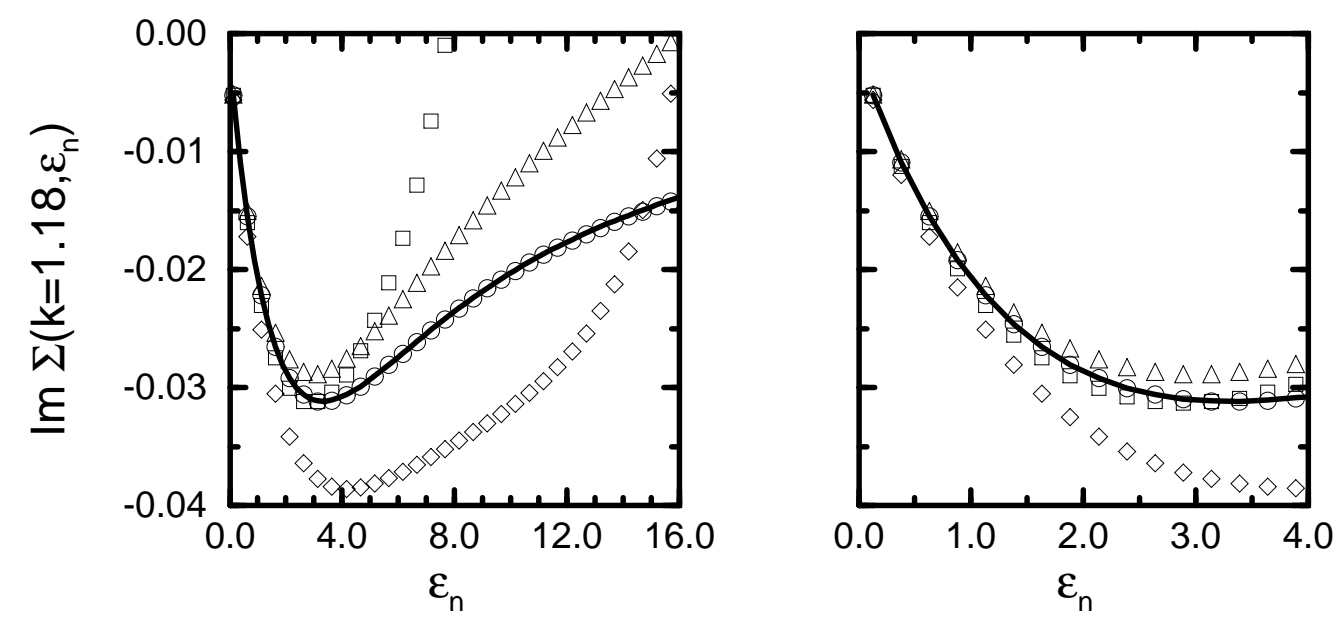

Figure 3: The second-order self-energy calculated using $G_{0}$ for the $1 \mathrm{D}$ Hubbard model with $U=t, \mu=1.4$, and $T=0.04$. The exact result (solid line), is compared with this method (o), the sharp cut-off method ( $\square$ ), the $\tau$-scheme $(\triangle)$, and the $\varepsilon_{n}$-scheme $(\diamond)$. For all frequencies this method leads to significantly more accurate results than the traditional frequency cut-off schemes. Note that every other point is plotted in the left panel for greater clarity.

at $\tau=0$. The optimal parameter choices for each function are again determined by requiring that the leading discontinuities in products such as the second-order self-energy are contained entirely in analytic terms. This requirement is satisfied if the $x_{i}(\mathbf{r})$ are chosen so that the discontinuities and the values of $G(\mathbf{r}, \tau), G^{\prime}(\mathbf{r}, \tau)$, etc. at $\tau=0$ are contained in analytic terms to the fullest extent possible with the available parameters.

For the full fluctuation exchange approximation, we introduce analogous asymptotic expansions for the particle-hole and particle-particle $T$-matrices, and again represent analytically the leading asymptotic behavior. The discontinuities of the $T$-matrix are determined by the discontinuities of the particle-hole and particle-particle bubbles, which are in turn determined by the single-particle Green's functions. The optimal parameters for the analytic part of the $T$-matrix are again chosen so that the leading discontinuities in the self-energy terms $\Sigma(\mathbf{r}, \tau)=T(\mathbf{r}, \tau) G(\mathbf{r}, \tau)$ are contained in the expressions which can be treated analytically, $\sigma(\mathbf{r}, \tau)=t(\mathbf{r}, \tau) g(\mathbf{r}, \tau)$. This requirement is satisfied if the values of $T(\mathbf{0}, 0), T^{\prime}(\mathbf{0}, 0)$, etc. are contained entirely in the analytic part of the t-matrix, $t(\mathbf{r}, \tau)$.

\section{Calculation of Thermodynamic Quantities}

Thermodynamic properties obtained from the grand thermodynamic potential calculated in a conserving approximation (such as the FEA) are guaranteed to be consistent with those obtained from a direct calculation involving the self-consistent Green's function and self-energy [6]. In general, thermodynamic properties and the grand thermodynamic potential are particularly sensitive to the high-frequency behavior of the propagator. A familiar example of the sensitivity to high-frequency parts of the Green's function is the 

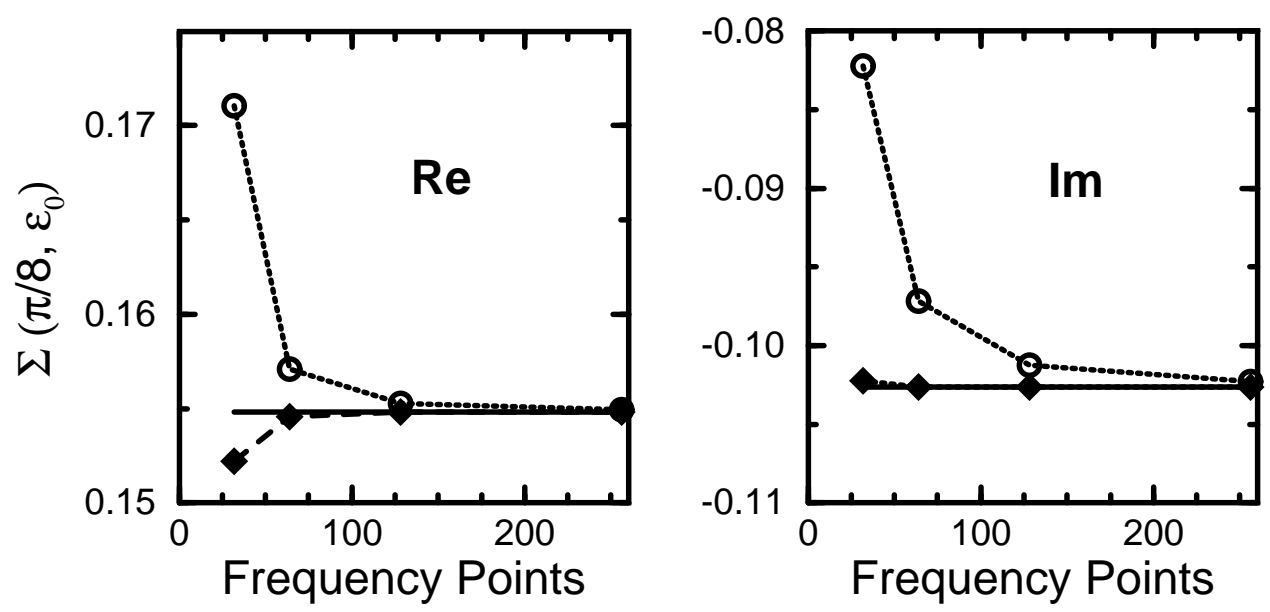

Figure 4: The self-consistent second-order self-energy at $\varepsilon_{0}$ for the 1D Hubbard model with $U=4 t, T=0.1$ and $\mu=-0.5$ as a function of the number of frequency points kept in the numerical part of $G$. The rate of convergence with respect to the number of frequency points obtained with this method (filled diamonds) is substantially improved with respect to the ordinary $\tau$-scheme (open circles). The solid line represents the $\varepsilon_{c} \rightarrow \infty$ limit of the ordinary $\tau$-scheme.

slowly converging frequency sum that results when the density is calculated by tracing the Green's function. It is not surprising that thermodynamic properties have proven difficult to calculate since changes in temperature produce only small relative changes in quantities like the free energy, which may be smaller than a fictitious temperature dependence introduced by the handling of the high frequency cut-off. In Fig. (5) we show that the calculation of the entropy can be achieved keeping a modest number of Matsubara frequencies when the method presented in this communication is used. Here, the entropy $S$ is computed two ways: (1) by numerically evaluating $S=-\partial F(T, N) /\left.\partial T\right|_{N}$ (open symbols) where $F$ is the Helmholtz free-energy, and (2) by evaluating $S=(E-F) / T$ where $E$ is the total energy (closed symbols). Again, this method (squares) produces more accurate results for a given number of frequency points than the $\tau$-scheme (as shown in the figure, the latter can even yield unphysical values of the entropy when a small number of frequency points are kept in frequency sums). Reliable thermodynamic calculations based on self-consistent perturbation theories may prove helpful in understanding the thermal properties of interacting quantum systems, especially since the small system sizes typical of exact methods make accurate calculations of thermodynamic properties problematic.

\section{Acknowledgments}

We thank A.Y. Liu for a critical reading of this manuscript. This work was supported in part by a grant of computer time from the DoD HPC Shared Resource Center, Naval Research Laboratory Connection Machine facility CM-5 / CM-200. 


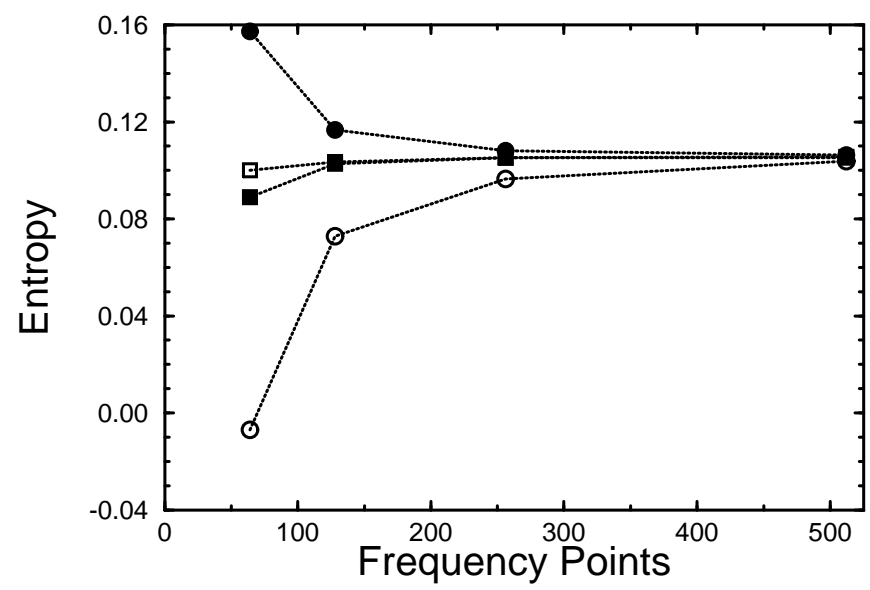

Figure 5: Self-consistent calculation for the entropy for the 3D Hubbard model with $16^{3}$ sites, $T=0.1, n=0.5$, and $U=4$ as a function of the number of frequency points. The open symbols are obtained using the formula $S=$ $-\partial F(T, N) /\left.\partial T\right|_{N}$ and the closed symbols from the formula $S=(E-F) / T$. The results for this method (squares) converge much more rapidly than those from the $\tau$-scheme (circles).

\section{References}

[1] J.W. Serene and D.W. Hess, in Electronic Structure and Mechanisms for High Temperature Superconductivity, edited by J. Ashkenazi, S.E. Barnes, F. Zuo, G. Vezzoli and B.M. Klein (Plenum, New York, 1991).

[2] J.W. Serene and D.W. Hess, Phys. Rev. B 44 , 3391 (1991).

[3] J.W. Serene and D.W. Hess, in Recent Progress in Many-Body Theories, Vol. 3, edited by T.L. Ainsworth et al. (Plenum, New York, 1992).

[4] P.G. McQueen, D.W. Hess and J.W. Serene, Phys. Rev. Lett. 70, 129 (1993).

[5] P.G. McQueen, D.W. Hess and J.W. Serene, Phys. Rev. B 50, in press.

[6] J.M. Luttinger and J.C. Ward, Phys. Rev. 118, 1417 (1960).

[7] G. Baym, Phys. Rev. 127, 1391 (1962).

[8] N.E. Bickers,D.J. Scalapino and S.R. White, Phys. Rev. Lett. 62, 961 (1989).

[9] A. Virosztek and J. Ruvalds, Phys. Rev. B 42, 4064 (1990).

[10] P.A. Lee and N. Read, Phys. Rev. Lett. 58, 2691 (1987).

[11] C.L. Kane, D.M. Newns, P.C. Pattnaik, C.C. Tsuei and C.C. Chi, in Electronic Structure and Mechanisms for High Temperature Superconductivity, edited by J. Ashkenazi, S.E. Barnes, F. Zuo, G. Vezzoli and B.M. Klein (Plenum, New York, 1991).

[12] D.W. Hess and J.W. Serene, J. Phys. Chem. Solids 52, 1385 (1991); and unpublished.

[13] C.M. Varma, P.B. Littlewood, S. Schmitt-Rink, E. Abrahams and A.E. Ruckenstein, Phys. Rev. Lett. 63, 1996 (1989). 
[14] J.J. Deisz, J.W. Serene and D.W. Hess, unpublished. It is important to note that our method of calculating the self-consistent staggered spin susceptibility is equivalent to solving an integral equation for the response function with irreducible vertices that consist of particle-particle and particle-hole bubble chains and Aslamosov-Larkin type diagrams.

[15] N.E. Bickers and S.R. White, Phys. Rev. B 43, 8044 (1991).

[16] R.T. Scalettar, D.J. Scalapino, R.L. Sugar and D. Toussaint, Phys. Rev. B 39, 4711 (1989).

[17] As an alternative to a sharp frequency cut-off, Pao and Bickers ( C.-P. Pao and N.E. Bickers, Phys. Rev. B 49, 1586 (1994)) ignore temperature renormalizations of Green's functions for $\varepsilon_{n}>\alpha T$ so that only a fixed number of the lowest frequency points are treated self-consistently as the temperature is lowered. 